\title{
In search of Neanderthals
}

Robin Dennell

Honor Among Thieves: A Zooarchaeological Study of Neandertal Ecology. By Mary C. Stiner. Princeton University Press: 1995. Pp. 447. \$69.50, £46.50. Mousterian Lithic Technology: An Ecological Perspective. By Steven L. Kuhn. Princeton University Press: 1995. Pp. 209. \$49.50, £39.50.

JUST occasionally, books on palaeoanthropology can be truly exciting. Here are two examples that break new ground in enhancing our understanding of Neanderthals and have important implications for how we study the behaviour of past populations. Both deal with the ancient coastal region of Latium, Italy, during the early Upper Pleistocene (35,000-100,000 years ago $(35-100 \mathrm{kyr}))$. The area is known mainly for its curious 'Pontian Mousterian', made largely of beach pebbles, and for Monte Circeo, an alleged (and undoubtedly spurious) site of Neanderthal ritual.

One of the biggest questions in palaeoanthropology concerns the emergence of modern humans. In Europe, the problem is tied to two events that happened $30-40$ kyr. First, Neanderthals either became extinct or evolved into fully modern humans (either way, there is no sign of them after 30 kyr). Second, and perhaps coincidentally, there is a transition from the Middle Palaeolithic period to the Upper Palaeolithic period around $35 \mathrm{kyr}$, marked primarily by the predominance of blade stone tools over flake tools, and the appearance of bone projectile points, hearths and art. The transition has (regrettably) dictated the course of most discussion on the emergence of modern humans. It is regrettable because it focuses attention only on the end of Mousterian culture (the final part of the Middle Palaeolithic) and not on its previous 100,000 years; there is no reason why this transition should have resulted in changes in anatomy, genetic make-up, subsistence, social behaviour or indeed anything except a limited range of technological changes; it also leads to the Mousterian being seen as monolithic rather than as a phenomenon with its own internal history and dynamics. This is grossly unfair to the earlier Neanderthals, who deserve to be studied in their own right as an extinct form of humanity.

The Mousterian itself is complex and has been studied in a bewildering number of ways. Continental archaeologists have tended to concentrate on stone tool technology and typology and, more recently, on the "châine operatoire" (the sequence in which flakes and blades were removed from the core of stone) and the life histories of artefacts and their function. British and US workers have relied more on geographical, geological and anthropological perspectives. Studies have ranged from individual sites to local regions (such as the Périgord in southwest France) and even larger climatic areas. Researchers studying animal remains from Upper Pleistocene sites (usually caves) have looked for changes or patterns in hunting strategies, using various methods that often predetermine the conclusions. Disagreements are rife, arising from differences in nationalities, professions, training and

\section{IMAGE UNAVAILABLE FOR COPYRIGHT REASONS}

prime-age animals - one of our unique adaptations as a predator. Her analysis is provocative in that she suggests that this change took place long before the Middle-Upper Palaeolithic transition 35 kyr and was accomplished without the use of bone- and stone-tipped projectiles.

Kuhn's concern is with the way in which Neanderthals maintained a supply of raw materials and artefacts as part of their survival strategy. His main findings match Stiner's. Before 55 kyr, Neanderthals were provisioning individuals with large renewable flake tools that could be re-sharpened while on scavenging trips; thereafter, the emphasis was on provisioning places in preparation for hunting prime-age animals, with toolmakers switching to the more economical technique of working cores and producing fresh blanks from local pebbles rather than re-sharpening old tools.

Before and after $55 \mathrm{kyr}$, Neanderthals obtained meat from dif-

ferent body parts and used different methods and toolkits for doing so. Kuhn shows that the changes were not accompanied by the appearance of new hunting items or methods of making stone tools; these changes would thus escape many continental researchers fixated on technologies or typologies.

The implications of all this are fascinating but unclear. Locally, the primary driving force behind the changes seems to be climatic: after $55 \mathrm{kyr}$, cave sites became further away from the coast owing to lowered sea levels. Because these climatic changes were global, other Neanderthal groups may have responded similarly. The books

inclinations of the researchers.

Both authors are well versed in evolutionary ecology and anthropology, aware of the need for testable hypotheses and prepared to treat the Mousterian as a phenomenon in its own right and not simply as a possible precursor of the Upper Palaeolithic. They examine the same sites (Mary Stiner concentrates on faunal remains, Steven Kuhn on artefacts); they bravely base their studies on excavations of several decades ago, when recording and collecting techniques were usually less stringent than today's; and they fully incorporate the past 20 years of research into their methodology.

Stiner looks at evolutionary ecology in terms of guilds of human and nonhuman predators (notably hyena and wolf) competing for the same prey (ungulates). Two patterns of Neanderthal subsistence emerge: the first, more than $55 \mathrm{kyr}$, is marked by the scavenging of head parts, perhaps to obtain fat in lean seasons; the second, later on, shows the ambushing of also raise important questions. Did Neanderthals use a wider range of techniques for obtaining meat from large animals than humans after $35 \mathrm{kyr}$ ? How and why did they ambush large animals without using hafted projectiles? Why didn't they often use fire for processing meat, as was common after $35 \mathrm{kyr}$ ? And, of course, why did they die out after being successful for so long?

These two remarkable books are landmark contributions that are outstanding in their theoretical and methodological rigour. Although they will remain largely inaccessible to nonspecialists, they should become role models for future related work. As the authors show so clearly, Neanderthals (and their predecessors) are fascinating in their own right, and not merely because the last of them may (or may not) have evolved into us.

Robin Dennell is in the Department of Archaeology and Prehistory, University of Sheffield, Sheffield S1 4ET, UK. 